\title{
Clonidina por Via Venosa na Técnica de Hipotensão Arterial Induzida para Timpanoplastias *
}

\section{Intravenous Clonidine in the Induced Arterial Hypotension Technique for Tympanoplasty}

Renato Mestriner Stocche, TSA ${ }^{1}$; Luiz Vicente Garcia, TSA ${ }^{2}$; Marlene Paulino dos Reis, TSA ${ }^{3}$; Oswaldo Miranda Junior ${ }^{4}$

\section{RESUMO}

Stocche RM, Garcia LV, Reis MP, Miranda Jr O - Clonidina por Via Venosa na Técnica de Hipotensão Induzida para Timpanoplastias

JUSTIFICATIVA E OBJETIVOS: A hipotensão arterial induzida é uma técnica eficaz para diminuir o sangramento durante atos cirúrgicos. A clonidina é um $\alpha_{2}$-agonista de ação central que já se mostrou segura em anestesia. O objetivo deste estudo foi verificar a eficiência da clonidina por via venosa como droga principal na hipotensão arterial controlada.

MÉTODO: Participaram do estudo prospectivo e duplamente encoberto, 36 pacientes de ambos os sexos, estado físico ASA I e II, divididos aleatoriamente em três grupos de 12 pacientes que receberam medicação pré-anestésica: clonidina $3 \mu \mathrm{g} \cdot \mathrm{kg}^{-1}$ (C3), clonidina $5 \mu \mathrm{g} \cdot \mathrm{kg}^{-1}$ (C5) ou solução fisiológica a $0,9 \%$ (Controle) 15 minutos antes da indução anestésica. A manutenção anestésica foi feita com isoflurano até a concentração máxima de $2 \%$. Foram anotados a PA e a FC antes, com 1 e 5 minutos após a indução e a cada 5 minutos de anestesia. Pacientes há mais de 15 minutos recebendo isoflurano a $2 \%$ e que não apresentaram PAS menor que 80 $\mathrm{mmHg}$ receberam nitroprussiato de sódio para indução da hipotensão arterial.

RESULTADOS: Três pacientes (25\%) no grupo C3, um (8\%) no grupo C5 e oito (66\%) no grupo controle necessitaram de nitroprussiato de sódio. A dose total de nitroprussiato para se induzir hipotensão arterial no grupo controle foi maior do que nos grupos C3 e C5 $(p<0,01)$. A incidência de complicações foi semelhante nos três grupos.

CONCLUSÕES: A clonidina por via venosa pode levar à hipotensão arterial induzida em cirurgias de timpanoplastias utilizando-se técnica de anestesia balanceada com concentração de isoflurano limitada em $2 \%$. Nas condições deste estudo, a clonidina não influenciou a qualidade anestésica e o tempo de despertar.

Unitermos: ANALGÉSICOS: clonidina; CIRURGIA, Otorrinolaringológica: timpanoplastia; HIPOTENSÃO CONTROLADA

\footnotetext{
* Recebido do (Received from) CET/SBA do Hospital das Clínicas da Faculdade de Medicina de Ribeirão Preto da Universidade de São Paulo (HC-FMRP-USP), Ribeirão Preto

1. Médico Assistente do Serviço de Anestesiologia do HC-FMRP-USP

2. Professor Assistente Doutor da Disciplina de Anestesiologia da FMRP-USP

3. Professora Associada da Disciplina de Anestesiologia da FMRP-USP

4. EX-ME do CET/SBA do HC da FMRP-USP

Endereço para correspondência (Correspondence to)

Dr. Renato Mestriner Stocche

Rua Adolfo Serra, 237 Alto da Boa Vista

14025-520 Ribeirão Preto, SP

E-mail: rstocche@keynet.com.br

Apresentado (Submitted) em 24 de julho de 2002

Aceito (Accepted) para publicação em 05 de dezembro de 2002

(c) Sociedade Brasileira de Anestesiologia, 2003
}

\section{SUMMARY}

Stocche RM, Garcia LV, Reis MP, Miranda Jr O - Intravenous Clonidine in the Induced Arterial Hypotension Technique for Tympanoplasty

BACKGROUND AND OBJECTIVES: Induced arterial hypotension is an effective technique to decrease surgical bleeding. Clonidine is an $\alpha_{2}$-agonist with central action which was proven to be safe in anesthesia. This study aimed at evaluating the efficacy of intravenous clonidine as the primary drug for induced arterial hypotension.

METHODS: Participated in this prospective double-blind study 36 patients of both genders, physical status ASA I and II, who were randomly distributed in three groups of 12 patients receiving the following preanesthetic medication 15 minutes before anesthetic induction: $3 \mu \mathrm{g} \cdot \mathrm{kg}^{-1}$ clonidine (C3), $5 \mu \mathrm{g} \cdot \mathrm{kg}^{-1}$ clonidine (C5) or $0.9 \%$ saline solution (Control). Anesthesia was maintained with isoflurane in a maximum concentration of $2 \%$. BP and HR were recorded before, 1 and 5 minutes after induction and at every 5 minutes of anesthesia. Patients receiving $2 \%$ isoflurane for more than 15 minutes and not presenting SBP below $80 \mathrm{mmHg}$ were administered sodium nitroprusside to induce arterial hypotension.

RESULTS: Sodium nitroprusside was needed in three C3 group patients (25\%), one C5 group patient (8\%) and eight control group patients (66\%). Total nitroprusside dose to induce arterial hypotension in the control group was higher as compared to groups C3 and C5 $(p<0.01)$. The incidence of complications was similar among groups.

CONCLUSIONS: Intravenous clonidine may lead to induced hypotension during tympanoplasties under balanced anesthesia with isoflurane concentration limited to $2 \%$. In the conditions of this study, clonidine has not affected anesthetic quality and emergence time.

Key Words: ANALGESICS: clonidine; CONTROLLED HYPOTENSION; SURGERY, Othorinolaringological: tympanoplasty

\section{INTRODUÇÃO}

A s cirurgias otorrinolaringológicas são, em sua grande Amaioria, realizadas em campos cirúrgicos ínfimos, tais como cavidades nasais, seios da face, conduto auditivo e ouvido médio. Pequenos sangramentos podem dificultar tecnicamente o ato cirúrgico, prolongando o tempo de cirurgia e, até mesmo comprometendo o resultado final ${ }^{1}$. Para tanto, técnicas anestésicas de hipotensão arterial induzida e controlada, têm sido amplamente empregadas com o intuito de diminuir o sangramento, proporcionando melhor campo cirúrgico ${ }^{2}$. Deve-se, entretanto, sempre respeitar as contra indicações à técnica, tais como: pacientes maiores de 60 anos, com hipertensão arterial de difícil controle, doença coronariana, diabetes, acidente vascular cerebral, insuficiência cardíaca e renal. 
Várias técnicas anestésicas têm sido empregadas na hipotensão arterial induzida. O isoflurano apresenta as vantagens de preservar a auto-regulação do fluxo e a oferta de oxigênio cerebrais ${ }^{3}$. Entretanto, concentrações elevadas podem ser necessárias, podendo levar à taquicardia reflexa, disritmias e prolongar o tempo de despertar ${ }^{4}$. Os vasodilatadores como o nitroprussiato de sódio e a nitroglicerina, bem como os $\beta$-bloqueadores, os bloqueadores ganglionares e os $\alpha$-bloqueadores também têm sido utilizados ${ }^{5,6}$. Porém, todas estas drogas apresentam limitações relacionadas a efeitos colaterais, contra-indicações e toxicidade.

A clonidina é uma droga anti-hipertensiva, $\alpha_{2}$-agonista central, amplamente utilizada em pacientes com hipertensão arterial de difícil controle. Como medicação pré-anestésica, a clonidina tem sido utilizada apresentando as seguintes características: diminui a resposta ao estresse da cirurgia e da intubação traqueal ${ }^{7}$, promove estabilidade hemodinâmica no peri-operatório ${ }^{8}$, diminui de maneira significativa o consumo de opióides e de halogenados ${ }^{9}$, promove efeito ansiolítico e antissialogogo ${ }^{10}$, não apresenta metabólicos tóxicos e apresenta baixa incidência de efeitos colaterais ${ }^{11}$.

O uso de clonidina por via oral na técnica de hipotensão arterial induzida per-operatória foi objetivo de vários estudos 4,12-14. Entretanto, a variabilidade no tempo de absorção por esta via e a associação com outras drogas com ação hipotensora dificultam a análise de seu efeito isoladamente. Além disto, a administração por via oral não permite estabelecer adequadamente a dose conforme o peso.

O objetivo deste estudo foi avaliar a eficácia de duas doses diferentes de clonidina, por via venosa, como droga principal, na técnica de hipotensão arterial induzida e a sua influência no consumo de anestésicos, na qualidade da anestesia e no despertar.

\section{MÉTODO}

Este estudo controlado, com distribuição aleatória e duplamente encoberto, foi aprovado pela Comissão de Ética em Pesquisa e realizado no Hospital das Clínicas de Ribeirão Preto. Dele, participaram 36 pacientes classificados como estado físico ASA I ou II, com idades entre 16 e 50 anos e peso entre 50 e $90 \mathrm{~kg}$, de ambos os sexos, e que foram submetidos à timpanoplastia unilateral. Foram excluídos pacientes com contra-indicações de hipotensão arterial induzida, com história de complicações anestésicas, de alergia a anestésicos do protocolo, que apresentavam fatores de risco para sangramento ou que não concordaram em assinar o termo de consentimento pós-informação.

Os pacientes foram distribuídos aleatoriamente em 3 grupos: Grupo C3 $(\mathrm{n}=12)$, que recebeu clonidina $3 \mu \mathrm{g} . \mathrm{kg}^{-1}$; Grupo C5 $(\mathrm{n}=12)$ que recebeu clonidina $5 \mu \mathrm{g} . \mathrm{kg}^{-1}$ ou Grupo S, controle, $(n=12)$, que recebeu solução fisiológica a $0,9 \%$. Após o sorteio do grupo ao qual o paciente pertenceria, as soluções pré-anestésicas foram preparadas por um médico não envolvido com a pesquisa. O preparo das soluções foi realizado utilizando-se seringas de $10 \mathrm{ml}$, de maneira que o volume final fosse sempre $9 \mathrm{ml}$. No grupo C3, a solução conti- nha $30 \mu \mathrm{g} \cdot \mathrm{ml}^{-1}$ de clonidina, no grupo C5 continha $50 \mu \mathrm{g} \cdot \mathrm{ml}^{-1}$ e no grupo $S$, solução fisiológica a $0,9 \%$. Desta forma, o volume injetado foi de $1 \mathrm{ml} .10 \mathrm{~kg}^{-1}$ para qualquer paciente em qualquer grupo. As soluções foram administradas lentamente por via venosa, 15 minutos antes da indução anestésica.

Todos os pacientes foram monitorizados previamente à cirurgia com pressão arterial pelo método oscilométrico no membro superior direito, cardioscopia nas derivações $D_{\|} \mathrm{e}$ V5, oximetria de pulso e capnometria. Após pré-oxigenação com balão e máscara, a indução anestésica foi realizada com propofol $\left(1,91 \pm 0,35 \mathrm{mg} \cdot \mathrm{kg}^{-1}\right)$ até a perda do reflexo palpebral, alfentanil $\left(20 \mu \mathrm{g} . \mathrm{kg}^{-1}\right)$ em bolus seguido de infusão contínua de $0,5 \mu \mathrm{g} \cdot \mathrm{kg}^{-1} \cdot \mathrm{min}^{-1}$ e pancurônio $\left(0,1 \mathrm{mg} \cdot \mathrm{kg}^{-1}\right)$ em bolus. Após a indução anestésica e intubação traqueal, foi administrada uma mistura de $\mathrm{N}_{2} \mathrm{O}_{\text {e }} \mathrm{O}_{2}$ a $50 \%$ e isoflurano até $2 \%$ em vaporizador calibrado, através de ventilação mecânica com objetivo de manter a $\mathrm{P}_{\mathrm{ET}} \mathrm{CO}_{2}$ entre 35 e $45 \mathrm{mmHg}$. Foram verificados os dados vitais imediatamente antes da administração da medicação pré-anestésica, 1 e 5 minutos após, imediatamente antes da indução anestésica, 1 minuto após, seguido de medidas intermitentes a cada 5 minutos até a extubação. Anotaram-se as concentrações de isoflurano a cada 5 minutos e calculou-se o consumo final que foi dividido pelo tempo em minutos de anestesia. Depois da extubação foram obtidos os sinais vitais a cada 15 minutos na SRPA. Considerou-se hipotensão arterial induzida adequada, níveis de pressão arterial sistólica entre 70 a $80 \mathrm{mmHg}$. Nos pacientes que não apresentaram hipotensão arterial após 15 minutos recebendo a concentração máxima de isoflurano $(2 \%)$ foi utilizado nitroprussiato de sódio titulado até no máximo de $8 \mu \mathrm{g} \cdot \mathrm{kg}^{-1} \cdot \mathrm{min}^{-1}$. Foram anotadas a freqüência ea titulação máxima de nitroprussiato necessárias para induzir hipotensão arterial adequada em cada paciente. Durante a administração de nitroprussiato, a concentração de isoflurano foi mantida em $2 \%$.

Quando se iniciou a colocação do enxerto timpânico, foi interrompida a infusão de alfentanil, a administração do $\mathrm{N}_{2} \mathrm{O}$ e alterou-se o sistema respiratório para sem reinalação, fixando-se a concentração inspirada de isoflurano em $1 \%$, até o final da cirurgia. Foi verificado o tempo decorrido entre a interrupção do isoflurano (término da cirurgia) e a abertura espontânea dos olhos. Também foi anotado o tempo entre a interrupção do isoflurano e a extubação traqueal, sendo que esta seria realizada quando o paciente apresentasse ventilação espontânea e rítmica durante pelo menos 30 segundos, mantendo a $\mathrm{P}_{\mathrm{ET}} \mathrm{CO}_{2}$ abaixo de $50 \mathrm{mmHg}$ e respondendo a estímulos verbais.

Ao término da cirurgia, a qualidade do campo cirúrgico em relação ao sangramento foi avaliada pelo cirurgião principal, através de escala analógica visual de 0 a 10 centímetros. Zero correspondendo ao pior campo possível (que impossibilitava a cirurgia) e dez correspondendo ao melhor campo possível, sem sangramento.

Afreqüência de eventos adversos foram anotadas. Considerou-se taquicardia a freqüência cardíaca (FC) maior que 100 bpm, bradicardia a FC menor que 50 bpm e hipotensão arterial excessiva a pressão arterial sistólica menor que 70 ou pres- 
são arterial média menor que $50 \mathrm{mmHg}$. Na SRPA foram anotados os eventos adversos e o tempo decorrido para a recuperação dos pacientes (nota 10 pelos critérios de Aldrete-Kroulik). Foi verificada também a necessidade de analgésicos nas primeiras 24 horas.

Os dados foram expressos em freqüência, freqüência relativa $(\%)$ ou médias \pm DP. As variáveis expressas em freqüência foram analisadas estatisticamente pelos testes do Qui-quadrado ou de Exato de Fisher. Dados expressos em médias foram analisados pelos testes de Mann-Whitney ou Análise de Variância para variáveis repetidas.

\section{RESULTADOS}

A distribuição nos grupos segundo os dados demográficos foram semelhantes, como mostra a tabela I. Os tempos de anestesia e cirurgia, bem como os tempos decorridos entre o final da cirurgia e a abertura dos olhos, final da cirurgia e a extubação traqueal e o final da anestesia e a alta da SRPAestão demonstrados na tabela II. Não houve diferenças estatísticas entre os grupos em relação a estes dados analisados. Todos os pacientes apresentaram ventilação espontânea adequada ao término da cirurgia, não havendo necessidade de utilizar naloxona.

Tabela I - Dados Demográficos

\begin{tabular}{lccc}
\hline & Controle & C3 & C5 \\
\hline Idade $(\text { anos })^{*}$ & $25,6 \pm 10,7$ & $26,5 \pm 10,5$ & $28,4 \pm 14,5$ \\
Peso $(\mathrm{kg})^{*}$ & $58,3 \pm 9,9$ & $61,1 \pm 14,3$ & $58,5 \pm 8,6$ \\
Sexo & & & \\
$\quad$ Masculino & 4 & 2 & 6 \\
$\quad$ Feminino & 8 & 10 & 6 \\
\hline
\end{tabular}

* Valores expressos pela Média \pm DP

Tabela II - Parâmetros Avaliados (Média \pm DP)

\begin{tabular}{lccc}
\hline & Controle & C3 & C5 \\
\hline Tempo de cirurgia & $130,0 \pm 41,9$ & $119,0 \pm 37,5$ & $142,2 \pm 31,8$ \\
Tempo de anestesia & $137,0 \pm 21,0$ & $160,9 \pm 34,3$ & $168,6 \pm 32,49$ \\
Tempo de abertura dos olhos & $7,7 \pm 6,5$ & $7,4 \pm 1,7$ & $8,8 \pm 3,8$ \\
Tempo de extubação & $9,5 \pm 5,1$ & $8,6 \pm 2,7$ & $8,9 \pm 3,5$ \\
Tempo Aldrete-Kroulik 10 & $121,7 \pm 59,5$ & $117,0 \pm 59,0$ & $129.7 \pm 65,1$ \\
\hline
\end{tabular}

Em oito pacientes $(66,7 \%)$ do grupo $S$ foi necessário administrar nitroprussiato para indução de hipotensão arterial adequada, enquanto somente 3 pacientes (25\%) do grupo C3 e 1 paciente $(8,3 \%)$ do grupo $C 5$ receberam nitroprussiato (Figura 1$)(p<0,01)$. O consumo de isoflurano por minuto de anestesia também foi diferente entre o grupo $S$ e os Grupos C3 e C5 ( $p<0,01)$, não havendo diferença entre os grupos que receberam clonidina (Figura 2). O consumo total de nitroprussiato em miligramas foi maior no grupo $S(4,32 \pm$ $1,39)$ do que no grupo C3 $(0,56 \pm 0,35)$ e C5 $(0.59 \pm 0.59)$ (Figura 3).

Revista Brasileira de Anestesiologia

Vol. 53, No 4, Julho - Agosto, 2003

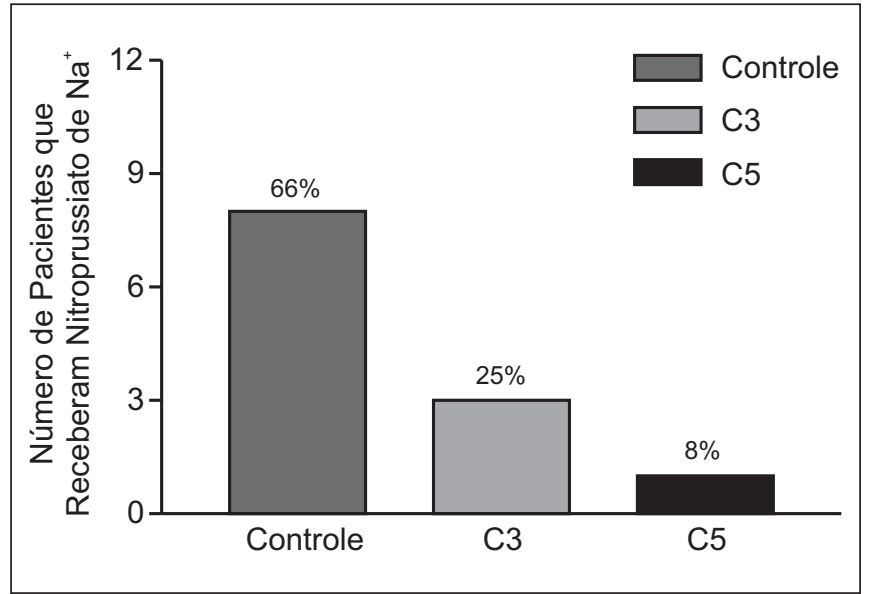

Figura 1 - Oito Pacientes no Grupo Controle, Três Pacientes no Grupo C3, e Um Paciente no Grupo C5 Necessitaram de Nitroprussiato de Sódio para levar a Hipotensão Arterial Induzida

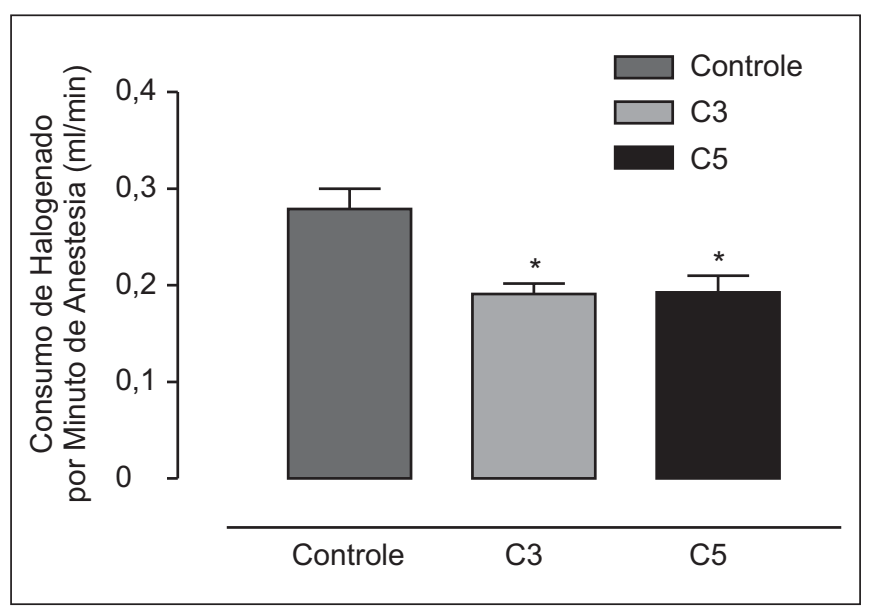

Figura 2 - Consumo de Isoflurano $\left(\mathrm{ml}^{\mathrm{min}} \mathrm{min}^{-1}\right)$ de Anestesia Dados Expressos em Média \pm DP: Controle 0,279 $\pm 0,073$ C3 0,191 $\pm 0,038$ e C5 0,193 $\pm 0,060\left(\mathrm{ml}_{\mathrm{min}} \mathrm{m}^{-1}\right)$

* Diferença estatística entre os grupos C3 e Controle, e C5 e controle pelo teste Mann-Whitney $(p<0,01)$

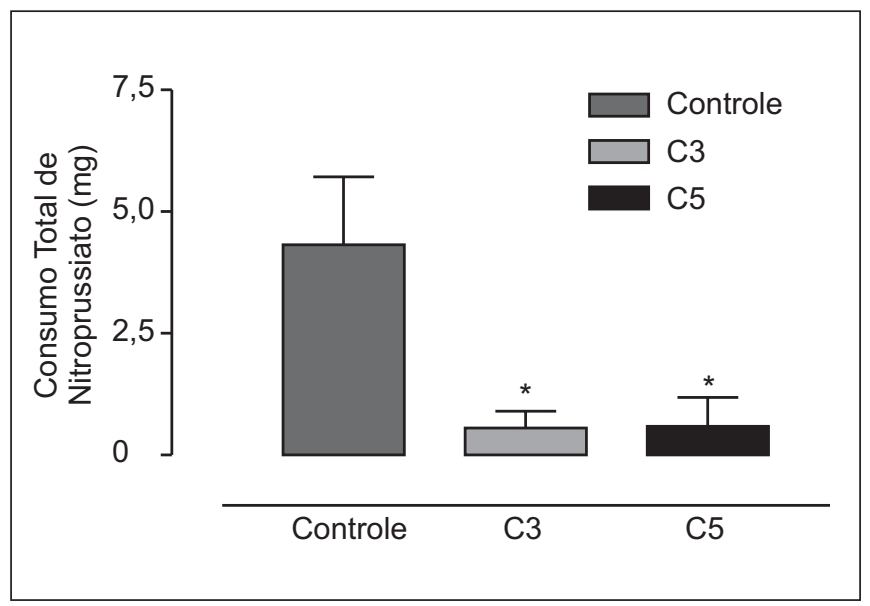

Figura 3 - Consumo de Nitroprussiato de Sódio (mg) Expressos em Média \pm DP: Controle 4,32 \pm 4,84, C3 0,56 $\pm 1,20$ e C5 0,59 \pm 2,05 (mg)

* Diferença estatística entre os grupos C3 e Controle, e C5 e controle pelo teste Mann-Whitney $(p<0,05)$ 
Os valores de pressão arterial sistólica (Figura 4) e pressão arterial média (Figura 5) foram semelhantes entre os grupos em todos momentos verificados. A freqüência cardíaca aumentou no grupo S quando comparada com grupo C3 e C5 (Figura 6). Entretanto, não houve diferença em relação à qualidade do campo cirúrgico avaliada pelo cirurgião, que apresentaram médias \pm DP para os grupos $S(8,3 \pm 0,48 \mathrm{~cm})$, C3 $(8,7 \pm 0,51 \mathrm{~cm})$ e C5 $(9 \pm 0,46 \mathrm{~cm})$.

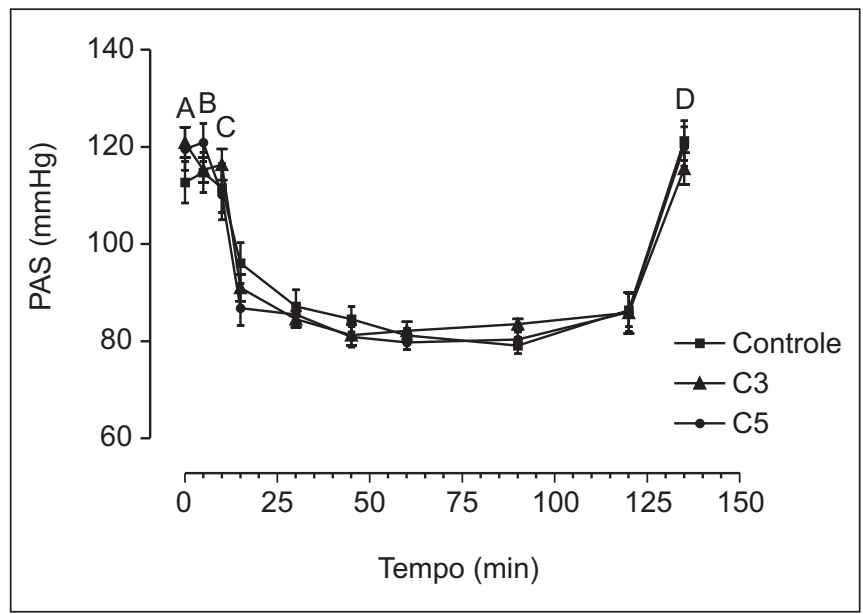

Figura 4 - Pressão Arterial Sistólica (Média \pm DP)

A - antes da medicação pré-anestésica, B - cinco minutos depois da medicação pré-anestésica, C - cinco minutos depois da indução anestésica e D - no final da anestesia

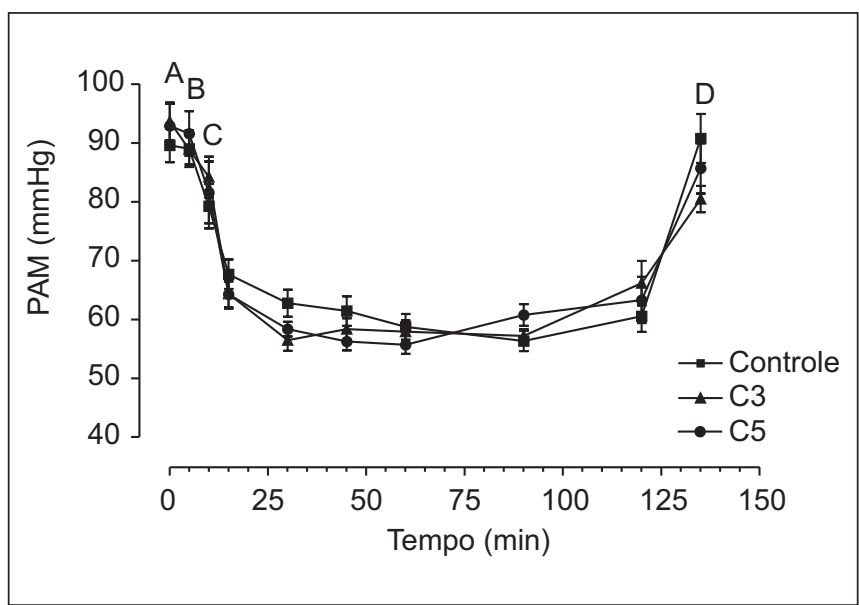

Figura 5 - Pressão Arterial Média (Média \pm DP)

A - antes da medicação pré-anestésica, B - cinco minutos depois da medicação pré-anestésica, $\mathbf{C}$ - cinco minutos depois da indução anestésica e D - no final da anestesia

A freqüência de eventos adversos foi maior no grupo $S$ em que ocorreram 10 episódios ( $p<0,01$ ). Houve diferença também entre os grupos $\mathrm{C} 3$ e $\mathrm{C} 5$ em que ocorreram, respectivamente, $6(50 \%)$ e $1(8,3 \%)$ eventos adversos $(p<0,01)$ (Tabela III).

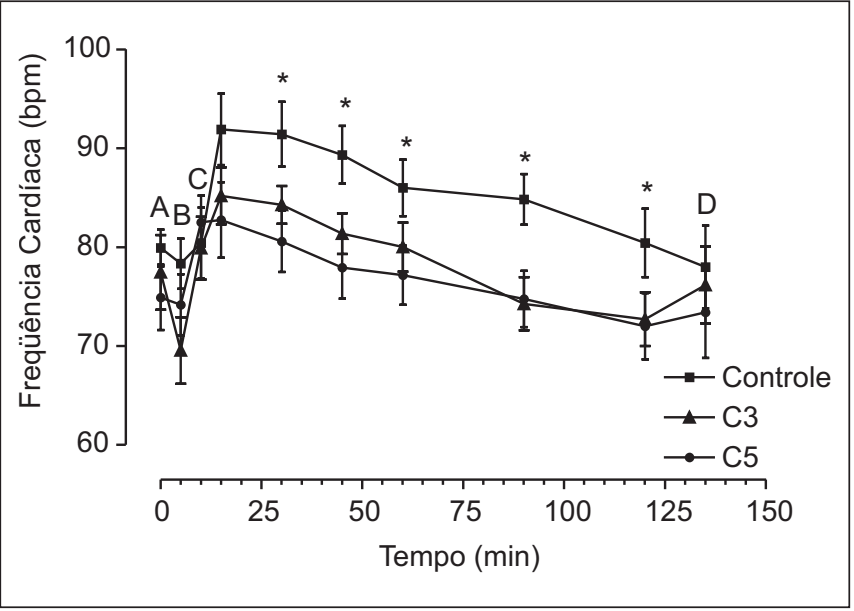

Figura 6 - Freqüência Cardíaca (Média $\pm D P$ )

A - antes da medicação pré-anestésica, B - cinco minutos depois da medicação pré-anestésica, C - cinco minutos depois da indução anestésica e $\mathbf{D}$ - no final da anestesia

* Diferença estatística entre os grupos S e C3 e C5 $(p<0,05)$

Tabela III - Eventos Adversos Ocorridos no Peri-Operatório

\begin{tabular}{lccc}
\hline & Controle & C3 & C5 \\
\hline Taquicardia & 3 & 0 & 0 \\
Bradicardia & 0 & 2 & 0 \\
Extrassístoles supraventriculares & 2 & 0 & 0 \\
Extrassístoles ventriculares & 1 & 0 & 0 \\
Hipotensão arterial excessiva & 1 & 1 & 1 \\
Agitação ao despertar & 0 & 1 & 0 \\
Náusea / vômitos & 0 & 2 & 0 \\
Resistência ao nitroprussiato & 3 & 0 & 0 \\
\hline
\end{tabular}

\section{DISCUSSÃO}

A clonidina, por diminuir o consumo de oxigênio corporal, pode ser vantajosa na técnica de hipotensão arterial induzida em que o equilíbrio entre a oferta e o consumo de oxigênio nos tecidos pode estar ameaçado ${ }^{15,16}$. Entretanto, a eficácia desta droga por via venosa, associada à técnica anestésica balanceada padronizada, em produzir hipotensão arterial controlada ainda não tinha sido testada.

Nossos resultados mostraram que a medicação pré-anestésica com clonidina por via venosa foi eficaz em promover hipotensão arterial controlada em cirurgias de timpanoplastias. No entanto, foram necessárias doses elevadas, pois com 3 $\mu \mathrm{g} \cdot \mathrm{kg}^{-1}, 25 \%$ dos pacientes necessitaram a associação com nitroprussiato contra $8,3 \%$, quando se utilizou $5 \mu \mathrm{g} \cdot \mathrm{kg}^{-1}$. Outros estudos procuraram estabelecer a utilidade da clonidina na técnica de hipotensão arterial induzida e encontraram resultados semelhantes. Aassociação de clonidina por via oral a hidroxizine, no pré-anestésico, pode ser útil na técnica de hipotensão arterial induzida ${ }^{12}$. Entretanto, neste estudo, a administração por via oral acarretou doses diferentes em relação ao peso do paciente, além de estudar a clonidina assoVol. 53, No 4, Julho - Agosto, 2003 
ciada a hidroxizine, não havendo controle com placebo ou comparação entre duas doses diferentes de clonidina, diferindo do nosso estudo. Em outro estudo, a clonidina foi efetiva como droga coadjuvante na técnica de hipotensão arterial induzida com labetalol. Neste estudo, a clonidina também foi administrada por via oral, o que proporcionou doses que variavam entre $3 \mathrm{e} 4 \mu \mathrm{g} . \mathrm{kg}^{-113}$. A associação de clonidina à prometazina e meperidina como medicações pré-anestésicas e labetalol na indução da hipotensão arterial durante anestesia venosa, também se mostrou eficaz ${ }^{14}$.

Diferindo desses outros estudos, o método empregado no nosso estudo permitiu avaliar a eficácia de duas doses diferentes de clonidina como medicação pré-anestésica em produzir hipotensão arterial controlada durante anestesia com isoflurano. O fato de limitar a concentração do isoflurano em $2 \%$ e padronizar todos os agentes anestésicos permitiu avaliar de maneira isolada a eficácia da clonidina em promover hipotensão arterial. Além do mais, permitiu comparar duas doses de clonidina com placebo. A via venosa apresenta as vantagens de adequar a dose em relação ao peso do paciente, bem como garantir concentrações adequadas da droga após poucos minutos de sua administração. Desta maneira, a droga pode ser injetada já no centro cirúrgico pelo próprio anestesista, tornando-se improváveis os problemas de dose ou horário de administração inadequados.

O objetivo de induzir hipotensão arterial controlada foi alcançado em todos os pacientes, variando-se somente a concentração do isoflurano e, se necessário, associando-se o nitroprussiato. Mais que isso, as médias das pressões arteriais foram semelhantes entre os grupos em todos os tempos verificados. Embora a FC tenha sido maior no grupo que não recebeu clonidina, não houve diferença na avaliação da qualidade do campo cirúrgico pelo cirurgião. O aumento da freqüência cardíaca no grupo $S$ pode ter sido reflexo do maior consumo de isoflurano e nitroprussiato neste grupo.

Da mesma forma que a clonidina potencializa os halogenados durante a anestesia, a concentração expiratória mínima para despertar (CAM-despertar) do isoflurano é significativamente menor quando se utiliza clonidina como medicação pré-anestésica ${ }^{17}$. O tempo decorrido do fim da cirurgia até a abertura ocular espontânea não foi alterado pelo uso de clonidina. Possivelmente, isto se deva ao menor consumo de isoflurano por minuto de anestesia apresentado nos grupos C3 e C5.

O tempo entre o fim da cirurgia e a extubação também não foi influenciado pela clonidina, o que está de acordo com outro estudo que não verificou influência desta droga no volume minuto, na freqüência respiratória e no volume corrente ${ }^{18}$. Apesar da clonidina potencializar o efeito e alterar a farmacocinética dos opióides ${ }^{19}$, nenhum paciente necessitou de naloxona para reverter depressão respiratória. O fato de termos usado o alfentanil, que apresenta menor tempo de eliminação, além de utilizarmos infusão contínua com doses relativamente baixas devem ter contribuído para este resultado. Afreqüência de eventos adversos foi maior no grupo que não recebeu clonidina devido, principalmente, ao maior consumo de isoflurano associado à maior necessidade de utili- zar-se nitroprussiato, traduzindo-se em maior freqüência de taquicardia e disritmias. Outros fatores que podem ter influenciado a menor freqüência de eventos adversos nos grupo C3 e C5 foi a própria ação da clonidina diminuindo os níveis de catecolaminas circulantes, reduzindo a ansiedade e a taquicardia resultante de alta concentração de isoflurano ${ }^{20}$. Em nosso estudo, o uso de clonidina produziu bradicardia intensa, diferindo de outros estudos. Aocorrência de bradicardia antes do início da cirurgia, encontrada em outro estudo semelhante, provavelmente se deva à associação de drogas sedativas, bem como ao maior tempo entre a medicação pré-anestésica e o início da cirurgia, levando à maior probabilidade de ocorrência de períodos sem estímulos ${ }^{12}$.

A recuperação anestésica através dos critérios de Aldrete-Kroulik não foi retardada pela clonidina. A alta da SRPA ocorreu em tempos equivalentes em todos os grupos. Aanalgesia pós-operatória proporcionada pela clonidina por via sistêmica é um assunto controverso ${ }^{21,22}$. Neste estudo, não encontramos diferença no consumo de analgésicos nas primeiras 24 horas entre os grupos.

Pelos dados obtidos, concluímos que a clonidina por via venosa pode levar à hipotensão arterial induzida e controlada em cirurgias de timpanoplastias utilizando-se técnica de anestesia balanceada com concentração de isoflurano limitada a $2 \%$. Nas condições deste estudo, a clonidina não influenciou a qualidade e o tempo de despertar, não prolongando o tempo de recuperação anestésica considerando-se os critérios de Aldrete-Kroulik.

\section{Intravenous Clonidine in the Induced Arterial Hypotension Technique for Tympanoplasty}

Renato Mestriner Stocche, TSA, M.D.; Luiz Vicente Garcia, TSA, M.D.; Marlene Paulino dos Reis, TSA, M.D.; Oswaldo Miranda Junior, M.D.

\section{INTRODUCTION}

ENT surgeries are performed, in their vast majority, in tiny surgical fields, such as nasal cavities, facial sinuses, auditory conduit and middle ear. Minor bleedings may technically impair surgery, prolonging its duration and even compromising outcomes ${ }^{1}$.

For such, anesthetic techniques with induced and controlled arterial hypotension have been widely used aiming at decreasing bleeding and providing better surgical fields ${ }^{2}$. However, counterindications should always be respected, such as patients above 60 years of age, with difficult to control arterial hypertension, coronary disease, diabetes, stroke, heart and renal failure.

Several anesthetic techniques have been used for induced arterial hypotension. Isoflurane has the advantage of pre- 
serving flow auto-regulation and brain oxygen supply ${ }^{3}$. However, high concentrations may be needed and may lead to reflex tachycardia and arrhythmias, in addition to delaying emergence ${ }^{4}$. Vasodilators, such as nitroprusside and nitroglycerin, as well as $\beta$-blockers, ganglial blockers and $\alpha$-blockers have been used ${ }^{5,6}$. All drugs, however, have limitations related to side-effects, counterindications and toxicity. Clonidine is an anti-hypertensive, central $\alpha_{2}$-agonist drug widely used in patients with difficult to control arterial hypertension. Clonidine has been used as preanesthetic medication and has the following profile: decreases surgical stress and tracheal intubation response ${ }^{7}$, promotes perioperative hemodynamic stability ${ }^{8}$, significantly decreases opioid and halogenate consumption ${ }^{9}$, promotes anti-anxiolytic and anti-sialogogue effects ${ }^{10}$, does not have toxic metabolites and has a low incidence of side-effects ${ }^{11}$.

Oral clonidine for perioperative induced hypotension has been subject to several studies ${ }^{4,12-14}$. However, the variability in absorption time through this route and the association with other drugs with hypotensive action impair the analysis of its isolated effects. In addition, oral administration does not allow the adequate formulation of the dose according to weight.

This study aimed at evaluating the efficacy of two different intravenous clonidine doses as the primary drug for induced arterial hypotension and its effect in anesthetic consumption, quality of anesthesia and emergence.

\section{METHODS}

After the Hospital das Clínicas, Ribeirão Preto Ethics Committee approval, participated in this controlled randomized and double blind study 36 patients of both genders, aged 16 to 50 years, weight 50 to $90 \mathrm{~kg}$, physical status ASA I or II who were submitted to unilateral tympanoplasty. Exclusion criteria were patients with counterindication for induced arterial hypotension, with previous history of anesthetic complications, allergic to standard anesthetics, presenting risk factors for bleeding or who have not agreed to sign their informed consent.

Patients were randomly distributed in three groups: Group C3 $(n=12), 3 \mu \mathrm{g} \cdot \mathrm{kg}^{-1}$ clonidine; Group C5 $(\mathrm{n}=12), 5 \mu \mathrm{g} \cdot \mathrm{kg}^{-1}$ clonidine; Group S, control ( $n=12), 0.9 \%$ saline solution. Preanesthetic solutions were prepared by a physician not involved with the study. Solutions were prepared in $10 \mathrm{ml}$ syringes in a way that the final volume was always $9 \mathrm{ml}$. Solution for group C3 had $30 \mu \mathrm{g} \cdot \mathrm{ml}^{-1}$ clonidine, for group C5 $50 \mu \mathrm{g} \cdot \mathrm{ml}^{-1}$ clonidine and for group S, $0.9 \%$ saline solution. This way, the volume injected was $1 \mathrm{ml} .10 \mathrm{~kg}^{-1}$ for all patients in any group. Solutions were slowly intravenously administered 15 minutes before anesthetic induction.

All patients were previously monitored with blood pressure by the oscilometric method in the right arm, cardioscopy in $D_{\|}$ and $V 5$ leads, pulse oximetry and capnometry. After pre-oxygenation with balloon and mask, anesthesia was induced with propofol $\left(1.91 \pm 0.35 \mathrm{mg} . \mathrm{kg}^{-1}\right)$ until loss of eyelid reflex, bolus alfentanil $\left(20 \mu \mathrm{g} \cdot \mathrm{kg}^{-1}\right)$ followed by $0.5 \mu \mathrm{g} \cdot \mathrm{kg}^{-1} \cdot \mathrm{min}^{-1}$ continuous infusion, and bolus pancuronium $\left(0.1 \mathrm{mg} \cdot \mathrm{kg}^{-1}\right)$. After anesthetic induction and tracheal intubation, a mixture of $50 \% \mathrm{~N}_{2} \mathrm{O} / \mathrm{O}_{2}$ and isoflurane up to $2 \%$ was administered through gaged vaporizer and mechanical ventilation to maintain $\mathrm{P}_{\mathrm{ET}} \mathrm{CO}_{2}$ between 35 and $45 \mathrm{mmHg}$.

Vital data were evaluated immediately before preanesthetic medication, 1 and 5 minutes after, immediately before anesthetic induction, 1 minute after and then at every 5 minutes until extubation. Isoflurane concentrations were recorded at 5-minute intervals and final consumption was calculated and then divided by the time in minutes of anesthesia. After extubation, vital signs were evaluated at 15 -minute intervals in the PACU.

Induced arterial hypotension was considered adequate when systolic blood pressure was between 70 and $80 \mathrm{mmHg}$. In patients without arterial hypotension after 15 minutes of maximum isoflurane concentration $(2 \%)$, titrated sodium nitroprusside was administered to a maximum of 8 $\mu \mathrm{g} \cdot \mathrm{kg}^{-1} \cdot \mathrm{min}^{-1}$. Frequency and maximum nitroprusside titration to induce adequate arterial hypotension were recorded for each patient. Isoflurane concentration was maintained in $2 \%$ during nitroprusside administration.

When tympanic grafting started, alfentanil infusion and $\mathrm{N}_{2} \mathrm{O}$ breathing were withdrawn and the respiratory system was changed to non-rebreathing system. Isoflurane inspired concentration was fixed in $1 \%$ until surgery completion. Time between isoflurane withdrawal (surgery completion) and spontaneous eye opening was recorded, in addition to time between isoflurane withdrawal and tracheal extubation, which was only achieved when patients presented spontaneous and rhythmic breathing for at least 30 seconds maintaining $\mathrm{P}_{\mathrm{ET}} \mathrm{CO}_{2}$ below $50 \mathrm{mmHg}$ and responding to verbal commands.

Surgical field quality in terms of bleeding was evaluated by the surgeon at surgery completion through the visual analog scale from 0 to 10 . Zero corresponded to the worst possible field (making surgery impossible) and 10 corresponded to the best possible field without bleeding.

The incidence of adverse effects was recorded. Tachycardia was defined as heart rate (HR) above $100 \mathrm{bpm}$, bradycardia was defined as HR below $50 \mathrm{bpm}$ and excessive arterial hypotension was defined as systolic blood pressure below 70 or mean blood pressure below $50 \mathrm{mmHg}$. Adverse effects and time for recovery (grade 10 according to Aldrete-Kroulik criteria) were recorded in the PACU. The need for analgesics in the first 24 hours was also evaluated.

Data are shown in frequency, relative frequency (\%) or mean \pm SD. Variables expressed in frequency were statistically analyzed by Chi-square or Fisher Exact tests. Data expressed in mean were analyzed by Mann-Whitney test or Analysis of Variance for repeated variables.

\section{RESULTS}

Demographics data were similar among groups as shown in table I. Anesthesia and surgery times, as well as time elapsed 
between: surgery completion and eye opening, surgery completion and extubation, and end of anesthesia and PACU discharge are shown in table II without statistical differences among groups. All patients had adequate spontaneous ventilation at surgery completion, with no need for naloxone.

Table I - Demographics Data

\begin{tabular}{cccc}
\hline & Control & C3 & C5 \\
\hline Age (years) & $25.6 \pm 10.7$ & $26.5 \pm 10.5$ & $28.4 \pm 14.5$ \\
Weight $(\mathrm{kg}){ }^{*}$ & $58.3 \pm 9.9$ & $61.1 \pm 14.3$ & $58.5 \pm 8.6$ \\
Gender & & & \\
Male & 4 & 2 & 6 \\
Female & 8 & 10 & 6 \\
\hline
\end{tabular}

* Values expressed in Mean \pm SD

Table II - Evaluated Parameters (Mean \pm SD)

\begin{tabular}{lccc}
\hline & Control & C3 & C5 \\
\hline Surgery duration & $130.0 \pm 41.9$ & $119.0 \pm 37.5$ & $142.2 \pm 31.8$ \\
Anesthesia duration & $137.0 \pm 21.0$ & $160.9 \pm 34.3$ & $168.6 \pm 32.49$ \\
Time for eye opening & $7.7 \pm 6.5$ & $7.4 \pm 1.7$ & $8.8 \pm 3.8$ \\
Time for extubation & $9.5 \pm 5.1$ & $8.6 \pm 2.7$ & $8.9 \pm 3.5$ \\
Time for Aldrete-Kroulik 10 & $121.7 \pm 59.5$ & $117.0 \pm 59.0$ & $129.7 \pm 65.1$ \\
\hline
\end{tabular}

Nitroprusside was needed in eight control group patients $(66.7 \%)$ to induce adequate arterial hypotension, while only three group C3 patients (25\%) and one group C5 patient $(8.3 \%)$ needed nitroprusside (Figure 1$)(p<0.01)$. Isoflurane consumption per minute of anesthesia was also different between control group and Groups C3 and C5 ( $<<0.01)$, without difference between groups receiving clonidine (Figure 2 ). Total nitroprusside consumption in milligrams was higher in control group (4.32 \pm 1.39$)$ as compared to groups C3 (0.56 \pm $0.35)$ and C5 (0.59 \pm 0.59$)$ (Figure 3$)$.

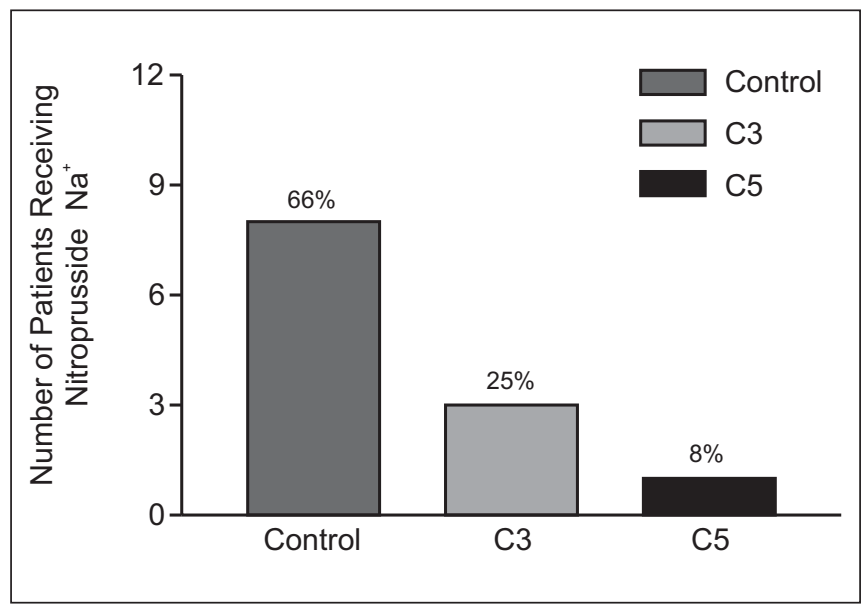

Figure 1 - Eight control group patients, three group C3 patients and one group C5 patient needed sodium nitroprusside to induce arterial hypotension

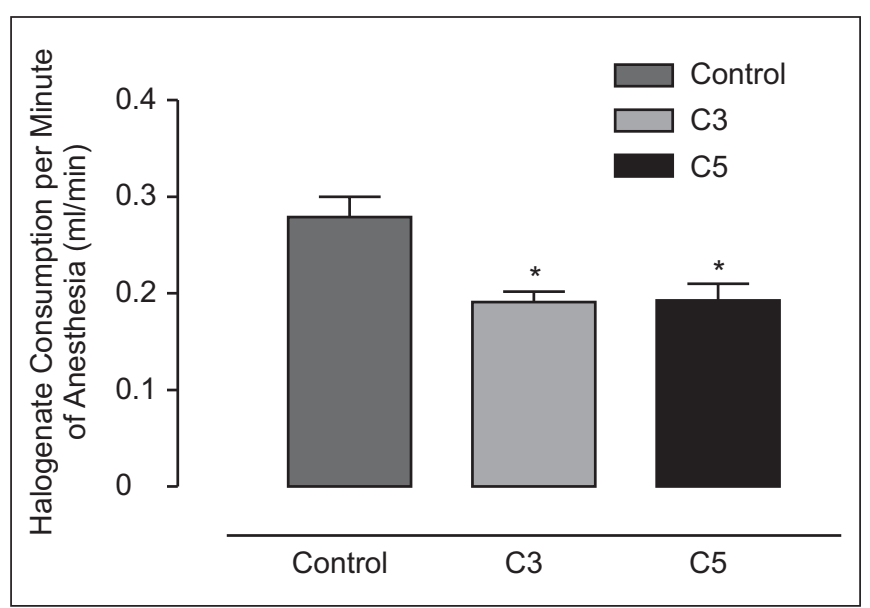

Figure 2 - Isoflurane Consumption ( $\mathrm{ml} . \mathrm{min}^{-1}$ )

Data in Mean \pm SD: Control $0.279 \pm 0.073$, C3 $0.191 \pm 0.038 \mathrm{e}$ C5 $0.193 \pm 0.060\left(\mathrm{ml} \cdot \mathrm{min}^{-1}\right)$

* Statistical difference between groups $\mathrm{C} 3$ and Control, and C5 and Control at Mann-Whitney test $(p<0.01)$

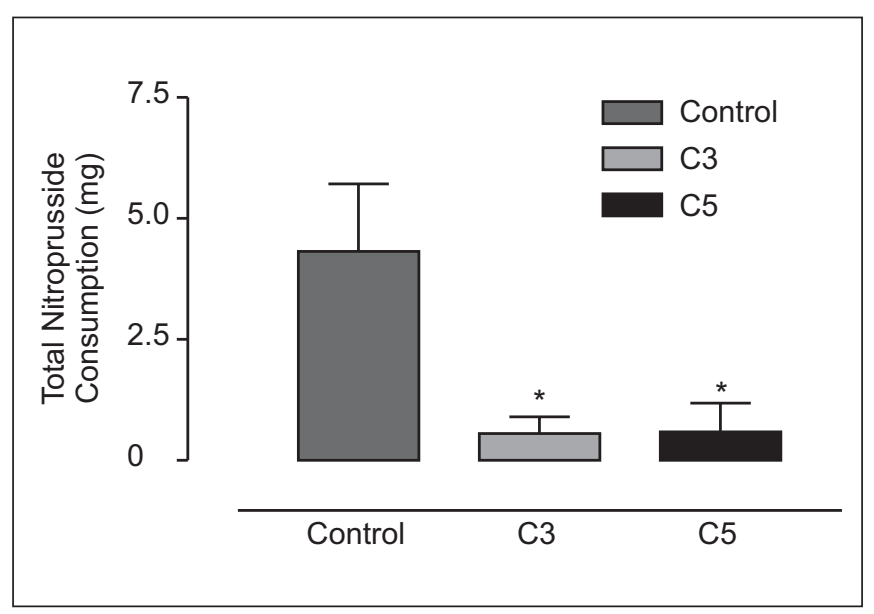

Figure 3 - Sodium Nitroprusside Consumption (mg) in Mean \pm SD Control $4.32 \pm 4.84$, C3 $0.56 \pm 1.20$ e C5 $0.59 \pm 2.05$ (mg)

* Statistical difference between groups C3 and Control, and C5 and Control at Mann-Whitney test $(p<0.05)$

Systolic blood pressure (Figure 4) and mean blood pressure (Figure 5) were similar among groups in all moments. Heart rate increased in control group as compared to groups C3 and C5 (Figure 6). There were, however, no differences in surgical field quality evaluated by the surgeon, which presented means \pm SD for groups $S(8.3 \pm 0.48 \mathrm{~cm}), C 3(8.7 \pm$ $0.51 \mathrm{~cm})$ and $\mathrm{C} 5(9 \pm 0.46 \mathrm{~cm})$.

The incidence of adverse events was higher in group $S$ with 10 episodes $(p<0.01)$. There have also been differences between groups C3 and C5 with $6(50 \%)$ and $1(8.3 \%)$ adverse events, respectively $(p<0.01)$ (Table III). 


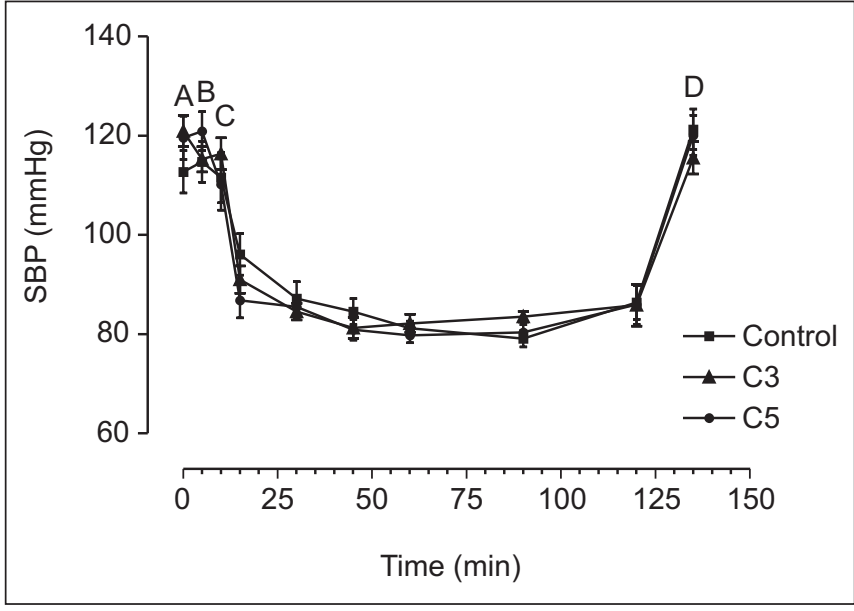

Figure 4 - Systolic Blood Pressure (Mean \pm SD)

A - before preanesthetic medication, $\mathbf{B}$ - five minutes after preanesthetic medication, $\mathbf{C}$ - five minutes after anesthetic induction and $\mathbf{D}$ - end of anesthesia

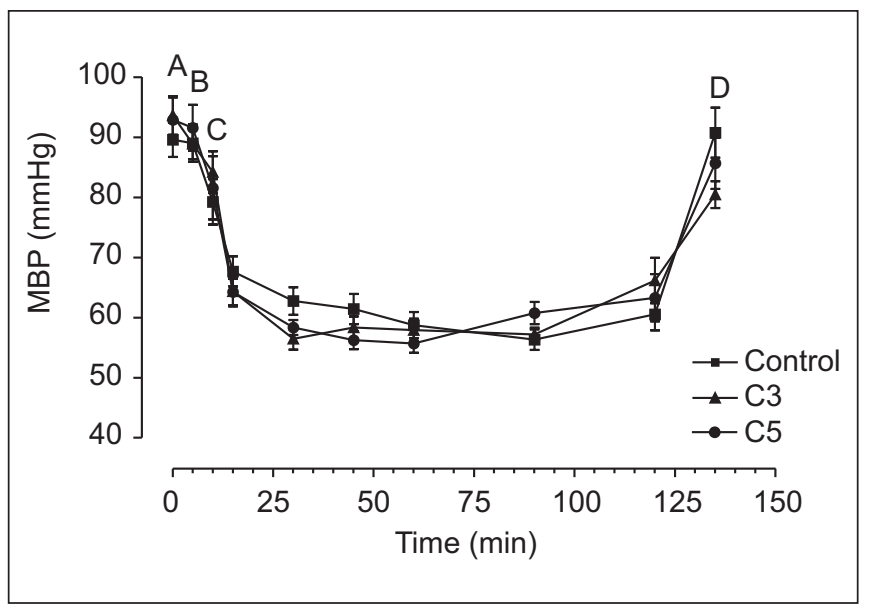

Figure 5 - Mean Blood Pressure (Mean \pm SD)

A - before preanesthetic medication, B - five minutes after preanesthetic medication, $\mathbf{C}$ - five minutes after anesthetic induction and $\mathbf{D}$ - end of anesthesia

Table III - Perioperative Adverse Events

\begin{tabular}{lccc}
\hline & Control & C3 & C5 \\
\hline Tachycardia & 3 & 0 & 0 \\
Bradycardia & 0 & 2 & 0 \\
Supraventricular extrasystole & 2 & 0 & 0 \\
Ventricular extrasystole & 1 & 0 & 0 \\
Excessive arterial hypotension & 1 & 1 & 1 \\
Agitation at emergence & 0 & 1 & 0 \\
Nausea / vomiting & 0 & 2 & 0 \\
Resistance to nitroprusside & 3 & 0 & 0 \\
\hline
\end{tabular}

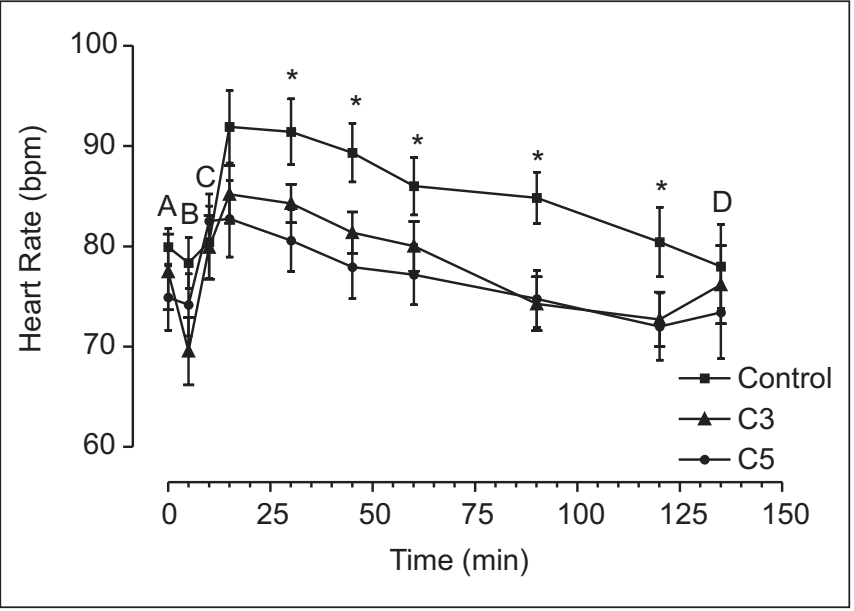

Figure 6 - Heart Rate (Mean \pm SD)

A - before preanesthetic medication, B - five minutes after preanesthetic medication, $\mathbf{C}$ - five minutes after anesthetic induction and $\mathbf{D}$ - end of anesthesia

* Statistical Difference between group S and groups C3 and C5 $(p<0.05)$

\section{DISCUSSION}

Clonidine, for decreasing body oxygen consumption, may be beneficial for induced arterial hypotension technique where the balance between tissue oxygen supply and consumption may be threatened ${ }^{15,16}$. However, the efficacy of this intravenous drug associated to the standardized balanced anesthesia technique to produce controlled induced arterial hypotension had not yet been tested.

Our results have shown that preanesthetic medication with intravenous clonidine was effective in promoting controlled arterial hypotension during tympanoplasties. High doses, however, were needed because with $3 \mu \mathrm{g} \cdot \mathrm{kg}^{-1}, 25 \%$ of patients needed the association with nitroprusside versus $8.3 \%$ when $5 \mu \mathrm{g} . \mathrm{kg}^{-1}$ were used. Other studies tried to establish the usefulness of clonidine in induced arterial hypotension and have found the same results. The association of oral clonidine to hydroxizine as preanesthetic medication may be useful for induced arterial hypotension ${ }^{12}$. However, in this study, oral administration has resulted in different doses related to patients' weight, in addition to studying clonidine associated to hydroxizine without placebo control or comparison between two different clonidine doses, thus differing from our study. In a different study, clonidine was effective as coadjuvant drug in induced arterial hypotension with labetalol. In this study, clonidine was also orally administered, leading to doses varying from 3 to $4 \mu \mathrm{g} \cdot \mathrm{kg}^{-113}$. The association of clonidine to prometazine and meperidine as preanesthetic medication and labetalol to induce hypotension during intravenous anesthesia has also shown to be effective ${ }^{14}$.

Differently from the above studies, the method used in our study allowed for the evaluation of the efficacy of two different preanesthetic clonidine doses to induce controlled arterial 
hypotension during anesthesia with isoflurane. The fact of limiting isoflurane concentration in $2 \%$ and standardizing all anesthetic agents has allowed for the isolated evaluation of the efficacy of clonidine in promoting arterial hypotension. In addition, this allowed the comparison of two clonidine doses with placebo. The intravenous route has the advantage of matching the dose to patients' weight, as well as assuring adequate drug concentrations few minutes after its administration. This way the drug may be injected already in the operating room by anesthesiologists themselves, thus making unlikely problems with inadequate dose or administration time. The purpose of inducing controlled arterial hypotension was achieved in all patients varying just isoflurane concentrations and, if needed, associating nitroprusside. Moreover, mean blood pressures were similar among groups in all moments. Although HR was higher in the group not receiving clonidine, there were no differences in the evaluation of surgical field quality by the surgeon. Increased HR in group $S$ could be a reflex of higher isoflurane and nitroprusside consumption.

In addition to potentiating halogenates during surgery, minimum isoflurane expiratory concentration for emergence (MAC-emergence) is significantly lower when clonidine is used as preanesthetic medication ${ }^{17}$. Time from surgery completion to spontaneous eye opening has not changed with clonidine. This might be due to lower isoflurane consumption per minute of anesthesia shown by groups C3 and C5.

Time between surgery completion and extubation was also not affected by clonidine, what is in line with a different study which has not evaluated the effects of this drug in minute volume, respiratory rate and tidal volume ${ }^{18}$. Although clonidine potentiates the effect and changes of opioid pharmacokinetics ${ }^{19}$, no patient needed naloxone to revert respiratory depression. The use of alfentanil, which has a faster elimination time, in addition to the administration in continuous infusion with relatively low doses might have contributed to such results.

The incidence of adverse events was higher in the group not receiving clonidine, especially due to higher isoflurane consumption associated to a higher need for nitroprusside and translated into a higher incidence of tachycardia and arrhythmia. Other factor which may have influenced the lower incidence of adverse events in groups C 3 and C5 was clonidine's action itself, decreasing circulating catecholamine levels, and anxiety and tachycardia resulting from high isoflurane concentrations ${ }^{20}$. In our study, clonidine has produced severe bradycardia, differing from other studies. Bradycardia before surgery found in a similar study was probably due to the association of sedative drugs, as well as to a longer time between preanesthetic medication and surgery, leading to a higher likelihood of periods without stimulation ${ }^{12}$.

Anesthetic recovery according to Aldrete-Kroulik criteria was not delayed by clonidine. Time for PACU discharge was similar for all groups. Postoperative analgesia induced by systemic clonidine is controversial ${ }^{21,22}$. Our study has not found differences in analgesic consumption in the first 24 postoperative hours among groups.
We have concluded from our data that intravenous clonidine may lead to induced and controlled arterial hypotension in tympanoplasties using balanced anesthesia with isoflurane concentration limited to $2 \%$. In the conditions of this study, clonidine has not affected emergence quality and time, not delaying anesthetic recovery time according to AldreteKroulik criteria.

\section{REFERÊNCIAS - REFERENCES}

01. Scholtes JL - Deliberate hypotension for middle ear microsurgery. Acta Anaesthesiol Belg, 1981;32:195-211.

02. Boisson-Bertrand D - Rédution du saignement en microchirurgie otorhinologique. Ann Fr Anesth Réanim, 1989,8:3A-4A.

03. Newman B, Gelb AW, Lam AM - The effect of isoflurane-induced hypotension on cerebral blood flow and cerebral metabolic rate for oxygen in humans. Anesthesiology, 1986;64:307-310.

04. Woodcock TE, Millard RK, Dixon J et al - Clonidine premedication for isoflurane-induced hypotension. Sympathoadrenal responses and a computer-controlled assessment of the vapour requirement. $\mathrm{Br} J$ Anaesth, 1988;60: 388-394.

05. Vieira JL, Katayama M - Hipotensão induzida com bloqueadores $\alpha$ e $\beta 1$. Halogenados: comparação entre enflurano, halotano e isoflurano em rinoplastias. Rev Bras Anestesiol, 1992;42: 201-206.

06. Grando TA, Puricelli E, Ishiguro RM et al - Avaliação do risco de complicações em duas técnicas de hipotensão controlada em cirurgia ortognática. Rev Bras Anestesiol, 1996;46:1-7.

07. Laurito CE, Baughman VL, Becker GL et al - The effectiveness of oral clonidine as a sedative/anxiolytic and as a drug to blunt the hemodynamic responses to laringoscopy. J Clin Anesth, 1991; 3:186-193.

08. Flacke JW, Bloor BC, Flacke WE et al - Reduced narcotic requirement by clonidine with improved hemodynamic and adrenergic stability in patients undergoing coronary bypass surgery. Anesthesiology, 1987;67:11-19.

09. Howie MB, Hiestand DC, Jopling MW et al - Effect of oral clonidine premedication on anesthetic requirement, hormonal response, hemodynamics, and recovery in coronary artery bypass graft surgery patients. J Clin Anesth, 1996;8:263-272.

10. Ramesh VJ, Bhardwaj N, Batra YK - Comparative study of oral clonidine and diazepan as premedicants in children. Int j Clin Pharmacol Ther, 1997;35:218-221.

11. De Kock M, Versailles $H$, Colinet B et al - Epidemiology of the adverse hemodynamic events occurring during "clonidine anesthesia": a prospective open trial of intraoperative intravenous clonidine. J Clin Anesth, 1995;7:403-410.

12. Welfringer P, Manel J, Garric J - Clonidine premedication and isoflurane anesthesia to reduce bleeding in otologic surgery. Ann Fr Anesth Reanim, 1992;11:125-311.

13. Toivonen J, Kaukinen S - Clonidine premedication: a useful adjunct in producing deliberate hypotension. Acta Anaesthesiol Scand, 1990;34:653-657.

14. Maroof M, Khan RM, Bhatti TH - Clonidine premedication for induced hypotension with total intravenous anaesthesia for middle ear microsurgery. Can J Anaesth, 1994;41:164-165.

15. Taittonen M, Kirvelä $O$, Aantaa R et al - The effect of clonidine and dexmedetomidine premedication on perioperative oxygen consumption and haemodynamic state. Br J Anaesth, 1997;78: 400-406. 
16. Stühmeier KD, Mainzer B, Cierpka J et al - Small, oral dose of clonidine reduces the incidence of intraoperative myocardial ischemia in patients having vascular surgery. Anesthesiology, 1996;85:706-712

17. Goyagi T, Tanaka M, Nishikawa T - Oral clonidine premedication reduces the awakening concentration of isoflurane. Anesth Analg, 1998;86:410-413.

18. Benhamou D, Veillette $Y$, Narchi $P$ et al - Ventilatory effects of premedication with clonidine. Anesth Analg, 1991;73:799-803.

19. Bernard J, Lagarde D, Souron R - Balanced postoperative analgesia: effect of intravenous clonidine on blood gases and pharmacokinetics of intravenous fentanyl. Anesth Analg, 1994; 79:1126-1132.

20. Tanaka S, Tsuchida $\mathrm{H}, \mathrm{Namba} \mathrm{H}$ et al - Clonidine and lidocaine inhibition of isoflurane-induced tachycardia in humans. Anesthesiology, 1994;81:1341-1349.

21. Mikawa K, Nishina K, Maekawa N et al - Oral clonidine premedication reduces postoperative pain in children. Anesth Analg, 1996;82:225-230.

22. Eisenach J, Hood DD, Curry R - Intrathecal, but not intravenous, clonidine reduces experimental thermal or capsaicin-induced pain and hyperalgesia in normal volunteers. Anesth Analg, 1998;87:591.

\section{RESUMEN}

Stocche RM, Garcia LV, Reis MP, Miranda Jr O - Clonidina por Vía Venosa en la Técnica de Hipotensión Inducida para Timpanoplastias

Justificativa y Objetivos: A hipotensión arterial inducida es una técnica eficaz para diminuir el sangramiento durante actos quirúrgicos. La clonidina es un $\alpha_{2}$-agonista de ación central que ya se mostró segura en anestesia. El objetivo de este estudio fue verificar la eficiencia de la clonidina por vía venosa como droga principal en la hipotensión arterial controlada.

Método: Participaron del estudio prospectivo y duplamente encubierto, 36 pacientes de ambos sexos, estado físico ASA I y II, divididos aleatoriamente en tres grupos de 12 pacientes que recibieron medicación pre-anestésica: clonidina $3 \mu \mathrm{g} . \mathrm{kg}^{-1}$ (C3), clonidina $5 \mu \mathrm{g} \cdot \mathrm{kg}^{-1}$ (C5) o solución fisiológica a 0,9\% (Control) 15 minutos antes de la inducción anestésica. La manutención anestésica fue hecha con isoflurano hasta la concentración máxima de 2\%. Fueron anotados la PA y la FC antes, con 1 y 5 minutos después de la inducción y a cada 5 minutos de anestesia. Pacientes a más de 15 minutos recibiendo isoflurano $2 \%$ y que no presentaron PAS menor que $80 \mathrm{mmHg}$ recibieron nitroprusiato de sodio para inducción de la hipotensión arterial.

Resultados: Tres pacientes (25\%) y el grupo C3, un (8\%) en el grupo C5 y ocho (66\%) en el grupo control necesitaron de nitroprusiato de sodio. La dosis total de nitroprusiato para inducir hipotensión arterial en el grupo control fue mayor de que en los grupos C3 y C5 $(p<0,01)$. La incidencia de complicaciones fue semejante en los tres grupos.

Conclusiones: La clonidina por vía venosa puede llevar a hipotensión arterial inducida en cirugías de timpanoplastia utilizándose técnica de anestesia balanceada con concentración de isoflurano limitada en $2 \%$. En las condiciones de este estudio, la clonidina no influenció la calidad anestésica y el tiempo de despertar. 